\title{
EVALUATION OF IMPROVEMENT IN RENAL FUNCTION AFTER FIRST-LINE CHEMOTHERAPY IN MULTIPLE MYELOMA PATIENTS PRESENTING WITH RENAL IMPAIRMENT
}

\author{
Syed Abdul Mannan Hamdani, Abdul Wahab, Musa Azhar, Faiza Iftikhar, Bushra Ahsan, Usman Ahmad
}

Shaukat Khanum Memorial Cancer Hospital, Lahore Pakistan

\begin{abstract}
Objective: To evaluate the effect of response to first-line chemotherapy on renal functions in multiple myeloma (MM) patients. Study Design: Cross-sectional study.

Place and Duration of Study: Shaukat Khanum Memorial Cancer Hospital, Lahore, from Jan 2013 to Dec 2017.

Methodology: Multiple myeloma patients with renal impairment (RI) at baseline were included. Data was collected from the hospital registry.The variables assessed pre and post first-line chemotherapywere age, gender, initial estimated glomerular filteration rate and serum creatinine levels. Progression-free survivaland overall survival (OS) was calculated for both groups of patients i.e those who had improvement in renal funtions and those who did not show improvement. Kaplan-Meier curves were drawn, and SPSS-20 was used for data analysis.

Results: Mean age was $48 \pm 7$ years. Out of 139 myeloma patients, 35 (25.1\%) had RI at baseline. Males were $23(65.7 \%)$, while females were $12(34.3 \%)$. Cyclophosphamide, thalidomide and deamethsone (CTD)were used in the majority 30 (85.7\%) followed by boretzomib, thalidomide and dexamethasone (VTD) in $2(5.7 \%)$ patients. Boretezomib and dexamethasone (BorDex), lenalidomide and dexamethasone (Len Dex), lenalidomde, cyclophosphamide and dexamethasone (LCD) were given to 1 $(2.8 \%)$ patient each. Serum creatinine levels normalized in $19(54.3 \%)$. Dialysis was required in $11(31.4 \%)$ patients out of which $3(8.6 \%)$ got dialysis-independent while $8(22.9 \%)$ patients remained dialysis-dependent. The medianoverall survival in whom creatinine levels normalized was 35 months while it was 15 months in those in whom it did not normalize.

Conclusion: Prompt administration of chemotherapy regimens can lead to an improvement in renal functions and ultimately have a substantialeffect on survival outcomes.
\end{abstract}

Keywords: Boretzomib and dexamethasone, Boretezomib, Cyclophosphamde, Cyclophosphamide and dexamethasone, Estimated glomerular filtration rate, Lenalidomide, Multiple myeloma, Renal impairment, Thalidomide and dexamethasone, Thalidomide and dexamethasone.

This is an Open Access article distributed under the terms of the Creative Commons Attribution License (https://creativecommons.org/licenses/by-nc/4.0/), which permits unrestricted use, distribution, and reproduction in any medium, provided the original work is properly cited.

\section{INTRODUCTION}

Multiple myeloma (MM) is aplasma cell neoplasmusually associated with presence of amonoclonal paraprotein (M protein). The most frequent clinical features includeanaemia, bone pains, fractures, cord compression, hypercalcemia, infections, and renal impairment ${ }^{1}$. It is a relatively uncommon malignancy accounting for $1-2 \%$ of all the cancers and $10-15 \%$ of all haematological cancers ${ }^{2}$. About $20-40 \%$ of patients of MM have renal impairment atpresentation, $10 \%$ of which require dialysis ${ }^{3,4}$. The kidney involvement at presentation mostly occurs in patients who have a high tumour burden, and it can lead to acute or chronic kidney disorder ${ }^{3}$. A variety of factors leadtorenal impairment (RI) in MM including light chain cast nephropathy (most common), hypercalcemia, light chain (AL) amyloidosis, light chain deposition disease, drug-induced renal damage, hyperviscosity and severe hyperurice-

Correspondence: Dr Syed Abdul Mannan Hamdani, Department of Medical Oncology, SKMCH Lahore Pakistan

Received: 26 Jul 2020; revised received: 23 Sep 2020; accepted: 09 Oct 2020 mia $^{4-6}$. The RI on presentation is associated with poor overall survival (OS), especially if there is a need for dialysis; however, reversal of renal functions back to normalcan lead to improvedsurvivals ${ }^{5,7}$.

Risk factors for developing RI include hypovolemia, diuretics use, hypercalcemia with or without nephrocalcinosis, repeated use of contrast agents, and drugs. Hypercalcemia can also lead to diabetes insipidus (DI) secondary to vasopressin resistance which in turn can lead to diuresis and hypovolemia and further aggravation of $\mathrm{RI}^{8}$.

The myeloma-related RI is a medical emergency which requires prompt initiation of treatment with adequate fluid replacement, discontinuation of nephrotoxic medication, correction of electrolytes and hemodialysis in selected patients $5,9,10$. Moreover, the early initiation of anti-myeloma therapy is the cornerstone of MM management. The neweragents thalidomide, lenalidomide, and bortezomib have become the standard of care in the last 10-15 years. Bortezomib based treatment has shown significant improvements in OS4. 
The objective of this study was which evaluated the improvement in renal functions in patients of MM who had RI at baseline. Although bortezomib based treat-ment is mostly used for treating patients of MM with RI; ourcentre functions in a resource-constrained environment and Thalidomide based regimens such as CTD (Cyclophos-phamide, thalidomide and dexamethasone) was mostly used as first-line treatment.

\section{METHODOLOGY}

This cross sectional anylysis was conducted at Shaukat Khanum Memorial Cancer Hospital, Lahore, form January 2013 to December 2017. Patient data was extracted from electronic hospital information system after obtaining approval from the institutional review board (EX-24-08-19-01). Informed consent was obtained from the patients in the cohort. The patient's age, laboratory parameters such as initial creatinine and eGFR, chemotherapy used, post-treatment creatinine and eGFR wererecorded in data analysis software. All the patients of MM who had renal impairment at baseline were included. The patients with prior history of chronic renal disease were excluded.

The International Myeloma Working Group (IMWG) criteria was used to describe the improvement in renal functions.Complete renal response was defined as attaining a consistent improvement ( $\geq 2$ months) in eGFR to $>60 \mathrm{ml} / \mathrm{min}$ from baseline. Partial response was an uninterrupted increase in eGFR from $<15 \mathrm{ml} /$ min to $30-59 \mathrm{ml} / \mathrm{min}$ and the minor response was a continual improvement in eGFR from $<15 \mathrm{ml} / \mathrm{min}$ to $15-29 \mathrm{ml} / \mathrm{min}$ or from $15-29$ to $30-59 \mathrm{ml} / \mathrm{min}$. Nil response was defined as a failure to meet the criteria mentioned above ${ }^{5}$. The response assessment was done after the end of first-line chemotherapy.

The Myeloma response assessment was done according to IMWG criteria. The stringent complete response was defined as a normal free light chain (FLC) ratio and absence of clonal cells in bone marrow by immunohistochemistry or immunofluorescence. Whereas, a complete response was defined as negative soft tissue plasmacytomas and $<5 \%$ plasma cells in the bone marrow. Very good partial response (VGPR) was defined as undetectable serum and urine $M$ proteins by electrophoresis, although they could be detected by immunofixation. The partial response (PR) was defined as $>50 \%$ reduction of serum $\mathrm{M}$ protein and reduction of urinary $\mathrm{M}$ protein by $90 \%$. The minimal response (MR) was $\geq 25 \%$, but $\leq 49 \%$ reduction in serum $M$ protein and $50-89 \%$ reduction in urinary $M$ protein, moreover, if plasmacytoma was present at baseline a $\geq 50 \%$ reduction was required in its size. Progressive disease (PD) was defined as an increase of $25 \%$ from lowest response value in any one or more of the following, i.e. serum $M$ component the absolute increase must be $>0.5 \mathrm{~g} / \mathrm{dl}$ or urine $\mathrm{M}$-component the absolute increase must be $>200 \mathrm{mg} / 24$ hours. In patients without measurable serum and urine $\mathrm{M}$ proteins, the difference between involved and uninvolved FLC levels, the absolute increase must be $>10 \mathrm{mg} / \mathrm{dl}^{11}$.

The OS and progression-free survival (PFS) were calculated for patients in whom creatinine levels normalized and in those they did not normalize. They were also assessed for patients who remained dialysisdependent and got free of dialysis.

SPSS-20 was used for statistical analysis of the data. Mean \pm standard deviation was used for continuous variables, while frequencies and percentages were reported for categorical variables. OS was defined as the time from date of diagnosis to the time of death of any cause or last follow-up. The Kaplan-Meier method was used to estimate survival as a function of time, and survival differences were analyzed by using the log-rank test. Statistical significance was defined as a two-tailed $p$-value $<0.05$.

\section{RESULTS}

During the study period 139 patients were diagnosed with MM, 35 of whomhad renal impairment at baseline. The median duration of follow up was 18 months. There were 23 (65.7\%) males and 12 (34.3\%) females. Pre-treatment and post-treatment lab parameters along with responses with regards to IMWG renal response criteria and myeloma response assessment are shown in table-I. Serum creatinine levels normalized in 19 patients while in 16it remainedabnormal. The relation between myeloma response and improvement in RI in terms of serum creatinine is shown in fig- 1. Among 16 patients who had VGPR, creatinine normalized in $12(75 \%)$ patients. Eight patients had PR, and creatinine normalized in 1 (12.5\%) patient. Out of 35 patients, $11(31.5 \%)$ required dialysis on the presentation, while $24(68.6 \%)$ did not require dialysis. Among the 11 patient who required dialysis, $3(27.3 \%)$ cameoff dialysis while $8(72.7 \%)$ remained dialysis-dependent. Overall, 16 (45.7\%) were alive, and 19 (54.3\%) were dead. In $30(85.7 \%)$ patients, CTD regimenwas used as first-line chemotherapy, while in 2 (5.7\%) VTD (Bortezomib, Thalidomide, Dexamethasone) was used. Lenalidomide/ Cyclophosphamide/Dexamethasone (LCD), Lenalido-mide/Dexamethasone (LenDex) and Bortezomib/Dexamethasone (BorDex) were used in one 
Table-I: Patient characteristics and laboratory parameters. CR: complete response, PR: partial response, VGPR: very good partial response, MR: minimal response, PD: progressive disease.

\begin{tabular}{|c|c|}
\hline Parameter & Value \\
\hline Age (years) & $48 \pm 7$ \\
\hline \multicolumn{2}{|l|}{ Gender } \\
\hline Male & $23(65.7 \%)$ \\
\hline Female & $12(34.3 \%)$ \\
\hline eGFR (ml/min) & Mean (range) \\
\hline Pre-treatment & $25(4-55)$ \\
\hline Post-treatment & $71(3.6-179)$ \\
\hline Serum Creatinine (mg/dl) & Mean \pm SD \\
\hline Pre-treatment & $4.3 \pm 3.4$ \\
\hline Post-treatment & $2.2 \pm 2.9$ \\
\hline \multicolumn{2}{|l|}{ Dialysis } \\
\hline Required & $11(31.5 \%)$ \\
\hline Not Required & $24(68.6 \%)$ \\
\hline $\begin{array}{l}\text { International myeloma working } \\
\text { group renal response criteria }\end{array}$ & n (\%) \\
\hline Complete response & $19(54.3 \%)$ \\
\hline Partial response & $8(22.9 \%)$ \\
\hline No response & $8(22.9 \%)$ \\
\hline $\begin{array}{l}\text { International Myeloma working } \\
\text { group criteria of Myeloma response } \\
\text { assessment }\end{array}$ & n $(\%)$ \\
\hline Very good partial response & $16(45.7 \%)$ \\
\hline Partial response & $8(22.9 \%)$ \\
\hline Minimal response & $4(11.4 \%)$ \\
\hline Progressive disease & $1(2.9 \%)$ \\
\hline Died during therapy & $4(11.4 \%)$ \\
\hline Lost to follow up & $2(5.7 \%)$ \\
\hline
\end{tabular}

patient $(2.9 \%)$ each. The OS at 60 months was 37\% for both groups of patients in whom creatinine levels normalized or remained abnormal. The median OS for patients in whom creatinine levels normalized and those in whom creatinine levels remained abnormal was 33 and 15 months respectively (fig-2A). The median PFS was 16 months for the entire cohort (fig-2B).
Among the patients who required dialysis initially, the median OS for those remaining dialysis-dependent and those coming off dialysis was 43 and 33 months respectively (fig-2C). As only three patients remained dialysis-dependent, the number is too small to draw any conclusions.

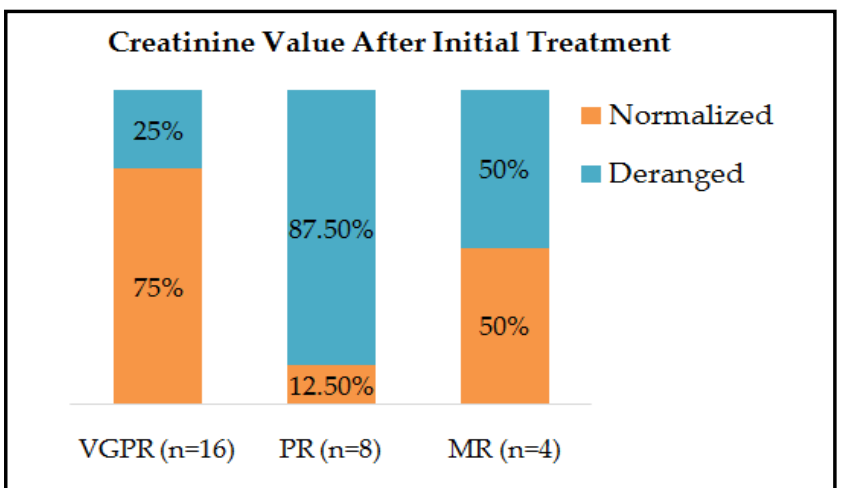

Figure: He relation between myeloma response and improvement in RI in terms of serum creatinine. VGPR: Very good partial response, PR: Partial response, MR: Minimal response.

\section{DISCUSSION}

Renal impairment is acommon complication of $\mathrm{MM}$, which has a direct impact on survival ${ }^{12}$. In this study, we evaluated the recovery in kidney functions and its effect on survival after using first-line chemotherapy, which in the majority of our patients was CTD regimen.

In western population, the reported median age at diagnosis of MM is 66 years9. In comparison, we noted that median age of our patientsat diagnosis was much lower i.e 48 years. Similar younger median age at the diagnosis is reported in some of the publications in our region $2,13,14$. There was male predominance i.e $65 \%$, which is also in line with what has been reported earlier ${ }^{15}$.
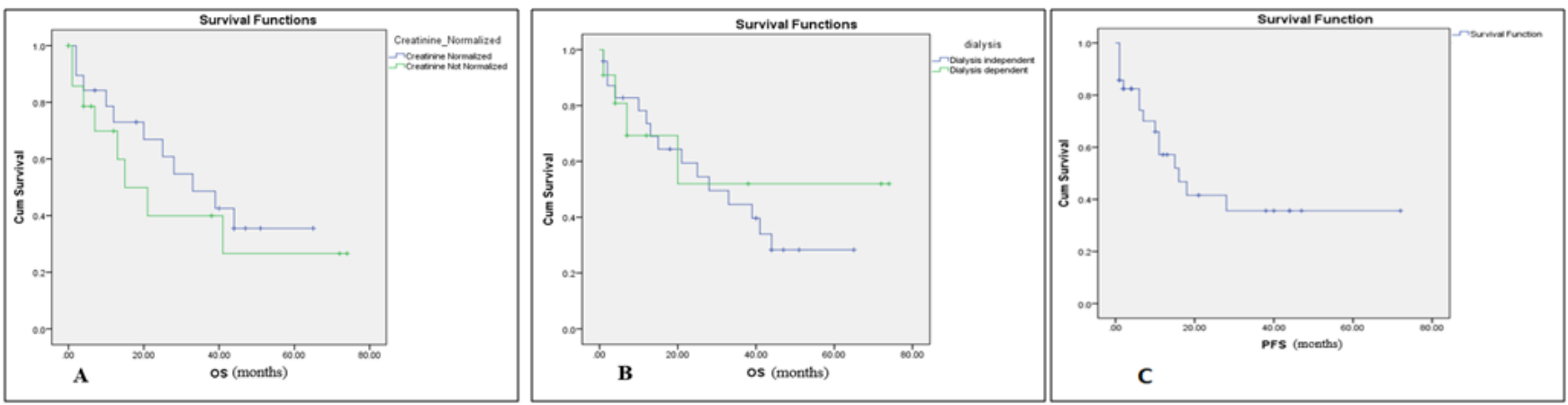

Figure-2A: Overall survival (OS) in reference to creatinine normalization. (B) Overall survival (OS) of patients receiving dialysis versus who got off dialysis. (C) Progression-free survival (PFS) of entire cohort. 
We noted that $25 \%$ of our patients presenting with MM had renal impairment. Most of the international data also reports similar figures of $20-40 \%$ of patients with MM presenting with RI. In addition, around $50 \%$ of MM patients advance to RI at some time during the disease course ${ }^{3}$.

There is considerable variability in the reported reversibilityof renal impairment. In our study, renal functions recovered in 19 (54.2\%) patients which is comparable to what is reported. Pönisch et al, for example, showed that there is $20-80 \%$ recovery of renal functions after starting chemotherapy ${ }^{16}$. The average time for reversibility of renal functions in study was 1.5 months, with two cycles of chemotherapy. This is also comparable to published data. For example, the time to reversibility of renal impairmentreported by Blade et al. is also around 1.5-1.6 months ${ }^{17}$.

We noted that 11 (31.5\%) of our patients required dialysis earlier in the course of the disease. Of these, $3(27.3 \%)$ cameoff from dialysis after first-line therapy, and remaining $8(72.7 \%)$ remained dialysis-dependent. The international data suggests that up to $10 \%$ of patients require dialysis at presentation, and death occurs in $30 \%$ of cases during the first two months of dialysis ${ }^{4}$. In our study, 2 out of $11(18 \%)$ patients died during the first two months of therapy. Although our reported early mortality is less than what has been reported, firm conclusions cannot be drawn as the sample size is small. The median OS of patients who remained dialysis-dependent was 43 months as compared to those patients who became dialysis independent (33 months). Again, due to small numbers, firm conclusions cannot be drawn. However, in a study by Kastritis et al. it was reported thatwith irreversible RI requiring permanent dialysis in MM, the median survival was about two years while $30 \%$ survived more thanthree years 4 . These findings suggest that those who have permanent renal impairment and become dialysis dependent, prolonged survivals can still be acheived ${ }^{4}$.

The treatment and outcomes of multiple myeloma have changed significantly since the advent of newer drugs namely bortezomib and thalidomide ${ }^{4,18}$. In our study, the median OS of patients with MM having RI on presentationwas 33 months for those in which creatinine levels normalized. Internationally before the year 2000, the median OS was 18-19 months for patients of MM with RI who had age $>65$ years. This median OS increased to 32 months after 2005. However, MM patients $<65$ years oldwith severe RI have had the best reported improvement in median OS of over 60 months ${ }^{18}$. This has been attributed mainly to the introduction of bortezomib which was not possible in our centre because of cost constraints as our hospital is charity based and offers free of cost treatment to majority of the patients. However, thalidomide based treatment was readily available.

Hence, the regimen most commonly used as the first line in our patients was CTD (30-85.7\% patients) patients, while only three patients receiving bortezomib based treatment as first-line.Bortezomib based treatments are reported to induce early CR and VGPR according to IMWG myeloma response assessment criteria ${ }^{19}$. Better PFS and OS have been reported when bortezomib based regimens are used as compared to CTD regimen. In one study, $78 \%$ of patients underwent autologous stem cell transplantation (ASCT) after bortezomib based VCD induction chemotherapy as compared to $48 \%$ when CTD was used ${ }^{19}$. Despite usage of CTD regimen in majority of our patients, comparable early CR and PR according to IMWG renal response assessment were seen.

\section{LIMITATION OF STUDY}

This study has a few limitations, first and foremost being limited number of patients. Secondly, due to cost and availability issues, the bortezomib based treatment was offered only a few patients. Therefore, the exact survival comparisons are not possible. The cytogenetic studies were not done in any of our patients at that time due to laboratory limitation. The cytogenetic studies not only give us an idea about standard, intermediate and high-risk categories but also help us in estimating survival, choosing induction chemotherapies and maintenance therapies ${ }^{9}$.

\section{CONCLUSION}

Prompt recognition and initiation of treatment, in patients of MM with RI, is of utmost importance as the prognosis of patients who get complete renal function recovery haveimproved survival significantly. Although regimens having bortezomib are now standard care for first-line treatment of MM especially with RI, regimens such as CTD provide a reasonablealternative with improved outcomes.

This study confirms that if treatment is initiated early, the RI in MM is reversible and patients have improved outcomes.

\section{CONFLICT OF INTEREST}

This study has no conflict of interest to be declared by any author. 


\section{REFERENCES}

1. Gastelum ZN, Biggs DM, Scott A. Multiple myeloma presenting as acute renal failure in the absence of other characteristic features. Cureus 2017; 9(9): e1703-08.

2. Sultan S, Irfan SM, Parveen S, Ali H, Basharat M. Multiple Myeloma: A retrospective analysis of 61 patients from a tertiary care center. Asian Pac J Cancer Prev 2016; 17(4): 1833-35.

3. Jung SH, Ahn JS, Yang DH, Cho MS, Kim JY, Ahn SY. Oliguria as an early indicator of mortality risk in patients with multiple myeloma and renal impairment. Blood Res 2015; 50(3): 167-72.

4. Kastritis E, Terpos E, Dimopoulos MA. Current treatments for renal failure due to multiple myeloma. Expert Opin Pharm 2013; 14(11): 1477-95.

5. Dimopoulos MA, Sonneveld P, Leung N, Merlini G, Ludwig H, Kastritis E, et al. International myeloma working group recommendations for the diagnosis and management of myeloma-related renal impairment. J Clin Oncol 2016; 34(13): 1544-57.

6. Faiman B, Tariman JD, Mangan PA, Spong J. Renal complications in multiple myeloma and related disorders: survivorship care plan of the IMF Nurse Leadership Board. Clin J Oncol Nurs 2011; 15(Supply-1): 66-69.

7. Heher EC, Rennke HG, Laubach JP. Kidney disease and multiple myeloma. Clin J Am Soc Nephrol 2013; 8(11): 2007-17.

8. Bozic B, Hölbling S, Völkel V, Sebesta C. Renal insufficiency in patients with multiple myeloma. Memo 2017; 10(3): 151-58.

9. Rajkumar SV, Kyle RA, editors. Multiple myeloma: diagnosis and treatment. Mayo Clin Proc; 2005: Elsevier.

10. Dimopoulos M, Kastritis E, Rosinol L, Blade J, Ludwig H. Pathogenesis and treatment of renal failure in multiple myeloma. Leuk 2008; 22(8): 1485-93.

11. Kumar S, Paiva B, Anderson KC, Durie B, Landgren O, Moreau $\mathrm{P}$, et al. International myeloma working group consensus criteria for response and minimal residual disease assessment in multiple myeloma. Lancet Oncol 2016; 17(8): e328-e46.
12. Laforet M, Jourde-Chiche N, Haddad F, Sallee M, Stoppa A, Brunet $\mathrm{P}$, et al. Evolution in the treatment of multiple myeloma and impact on dialysis independence: data from a French cohort from 1999 to 2014. Blood Cancer J 2016; 6(3): e409-412e.

13. Hameed A, Ali J, Munawar K, Arshad F, Badar F, Siddiqui N. Characteristics and outcomes of patients with multiple myeloma: Data from a developing country. Med J Islam Repub Iran 2018; 32(1): 1-5.

14. Lu J, Chen W, Huo Y, Huang X, Hou J. Clinical features and treatment outcome in newly diagnosed Chinese patients with multiple myeloma: results of a multicenter analysis. Blood Cancer J 2014; 4(8): e239-242e.

15. Weil C, Gelerstein S, Moser SS, Chodick G, Ben-David NB, Shalev V, et al. Real-world epidemiology, treatment patterns and survival of multiple myeloma patients in a large nationwide health plan. Leuk Res 2019; 85(2): 106219-23.

16. Pönisch W, Andrea M, Wagner I, Hammerschmidt D, Kreibich U, Schwarzer A. Successful treatment of patients with newly diagnosed/untreated multiple myeloma and advanced renal failure using bortezomib in combination with bendamustine and prednisone. J Cancer Res Clin Oncol 2012; 138(8): 1405-12.

17. Bladé J, Rosiñol L, Cibeira MT, Fernández de Larrea C. Treatment of relapsed myeloma in a patient with renal insufficiency. J Clin Oncol 2018; 36(20): 2012-16.

18. Dimopoulos M, Delimpasi S, Katodritou E, Vassou A, Kyrtsonis M, Repousis P, et al. Significant improvement in the survival of patients with multiple myeloma presenting with severe renal impairment after the introduction of novel agents. Ann Oncol 2014; 25(1): 195-200.

19. Vigolo S, Zuckermann J, Bittencourt RI, Silla L, Pilger DA. Comparison of cyclophosphamide-thalidomide-dexamethasone to bortezomib-cyclophosphamide-dexamethasone as induction therapy for multiple myeloma patients in Brazil. Hematol Oncol Stem Cell Ther 2017; 10(3): 135-42. 